\title{
The application of niobium and tantalum oxides for implant surface passivation
}

\author{
V.V. Starikov, ${ }^{1}$ S.L. Starikova, ${ }^{2}$ A.G. Mamalis, ${ }^{3}$ S.N. Lavrynenko ${ }^{1, *}$ and J.J. Ramsden ${ }^{4}$ \\ ${ }^{1}$ National Technical University “Kh.P.I. ”, Ukraine \\ ${ }^{2}$ Kharkov State Medical University, Ukraine \\ ${ }^{3}$ National Technical University of Athens, Greece \\ ${ }^{4}$ Department of Materials, Cranfield University, UK
}

\begin{abstract}
The corrosion of the surfaces of metallic implants is a very aggressive process, which can cause inflammation and deposition of metal in the surrounding live tissue. Prevention of corrosion and improvement of the functional characteristics of different coatings for application on implant surfaces is essential. Niobium and tantalum anodic oxides of optimal thickness have enabled the manufacture of a new generation of bioengineering products displaying minimal corrosion.
\end{abstract}

Keywords: anodic oxides, coating, implants, niobium, tantalum

\section{INTRODUCTION}

Despite the advantages of ceramics, with their high corrosion stability in vivo, most medical implant constructions are still made from metals [1]. To increase the corrosion stability of metals, different coatings are applied to the implant surfaces, typically such coatings are the oxides of the metals in the implants [2].

For an oxide film to have protective properties it must satisfy the following requirements:

- to be unbroken and pore-free;

- to have good adhesion with the metal;

- to have a thermal expansion constant near to the value for the metal;

- to be chemically inert in different environments;

- to be hard and have minimal wear under load.

The oxides of metals such as $\mathrm{Al}, \mathrm{Ti}, \mathrm{Zr}, \mathrm{Nb}$ and $\mathrm{Ta}$ satisfy all these properties to some degree [3-5]. Indeed, some of these metal oxides are used in medicine independently without a metal substrate, such as implant constructions from sapphire (a single-crystal modification of $\left.\mathrm{Al}_{2} \mathrm{O}_{3}\right)[6,7]$.

Titanium is the most widely used material for medical implant manufacture [8]. Its chemical passivity is provided by the oxide film $\left(\mathrm{TiO}_{2}\right)$, covering the entire free surface; a result of titanium contact with air. But titanium is unable to satisfy all the requirements necessary for an implant material because of its insufficient corrosion stability [9-11]. The application of combined implants consisting of a metal base and a ceramic coating also does not give a complete solution to the problem, because of the low adhesion strength and fragility of ceramic coatings $[12,13]$.

${ }^{*}$ Corresponding author. E-mail: lavr@kpi.kharkov.ua

\section{FEATURES OF NIOBIUM AND TANTALUM OXIDE COATINGS ON IMPLANTS AND OPTIMIZATION OF THEIR THICKNESS}

Electrochemical corrosion activity on titanium implants can be reduced by the use of other metals, in particular tantalum and niobium. These metals can also be used as the base material for implants when their surfaces are oxidized to increase corrosion stability [14]. It is possible to sputter tantalum or niobium oxide on the surface of other metals. Both lead to the metal's insulation from environmental stress by the unbroken, durable, and at the same time elastic, oxide. Nevertheless there is not sufficient information available to determine the optimum choice of oxidation type and the influence of oxide thickness. It has been established that an increase of oxide thickness on the implant metal surface results in more protein adsorption at the implantbone contact. However, the optimum thickness and the structure of oxide layer remains to be investigated [15].

Research into the electrochemical activity of niobium and tantalum in comparison with titanium was conducted for two cases: (1) in the initial state when the metal's surface was coated by its native oxide film; and (2) after passivation by anodic oxidation of its surface.

The electrochemical process is characterized by the electrode potentials of the metals, the voltage on the galvanopair and the short-circuit currents between metals. The second metal electrode was cobalt-chromium alloy (Co $62 \%$, Cr 30\%, Mo 5\%, C 0.4\%), which is widely used for implants and dental orthopaedic construction. The phenomena taking place during electrochemical corrosion requires all of these parameters to be considered to establish the corrosion and biocorrosion environment. The measurements were conducted in an electrochemical cell filled with physiological fluid $(0.9 \%$ aqueous solution of $\mathrm{NaCl}$ ) and an $\mathrm{AgCl}$ reference electrode was used. 
Model samples for the electrochemical tests were made of titanium, niobium and tantalum foils of thickness $t=5 \times 10^{-4} \mathrm{~m}$. Relaxation of mechanical stresses in the sample bulk was achieved by vacuum annealing at pressure $P=1 \times 10^{-3} \mathrm{~Pa}$ during $\tau=30 \mathrm{~min}$, which also cleaned and removed impurities from the samples. Titanium samples were annealed at temperature $T=950 \mathrm{~K}$ and niobium and tantalum at $T=2000 \mathrm{~K}$.

The influences of the oxidation method and oxide thickness on the passivation of niobium and tantalum were also investigated. Two oxidation techniques were used: thermal and anodic. In the first case the reaction between metal and oxygen occurred by a diffusive mechanism and the thickness of the oxide layer was determined by the exposure time of the heated metal $(T=670 \mathrm{~K})$ in air. Four sets of samples were prepared with exposure times $\tau=1,2,4$ and 8 hours. Anodic oxidation was conducted in an electrolyte $(0.01 \%$ aqueous solution of $\mathrm{H}_{3} \mathrm{PO}_{4}$ ), with a tantalum counterelectrode. The thickness of the oxide film $t_{\mathrm{ox}}$ on the sample surface was determined by the anodizing voltage $U_{\mathrm{a}}$ according to the formula

$$
t_{\mathrm{ox}}=\alpha_{\text {Metal }} U_{\mathrm{a}} \text {, }
$$

where $\alpha_{\text {Metal }}$ is the anodizing constant $\left(\alpha_{\mathrm{Ta}}=1.6 \times 10^{-9} \mathrm{~m} / \mathrm{V}\right.$; $\left.\alpha_{\mathrm{Nb}}=2.4 \times 10^{-9} \mathrm{~m} / \mathrm{V}\right)$. Seven voltages were used: $U_{\mathrm{a}}=30$, $45,60,75,90,105$ and $120 \mathrm{~V}$.

An indicator of corrosion activity is the electrode potential; the more negative the potential, the greater the activity. The data (Table 1) shows that the greatest activity is in the initial state. The oxide layer increases the passivity of the metal as shown by the potential shifts in the positive direction; the degree of titanium passivation surpassed that of niobium.

Table 1. Electrode potentials ${ }^{a}$ of implant metals.

\begin{tabular}{clr}
\hline Material & \multicolumn{1}{c}{ State } & \multicolumn{1}{c}{$E / \mathrm{V}$} \\
\hline $\mathrm{Ti}$ & initial state & -0.044 \\
& after oxidation & 0.325 \\
$\mathrm{Nb}$ & initial state & -0.005 \\
& after oxidation & 0.170 \\
$\mathrm{Ta}$ & initial state & 0.061 \\
& after oxidation & 1.940 \\
Cobalt-chromium alloy & & -0.101 \\
\hline
\end{tabular}

${ }^{a}$ With reference to $\mathrm{Ag} / \mathrm{AgCl}$.

The corrosion process occuring between the metal and a cobalt-chromium alloy counterelectrode was ambiguous (Table 2). The titanium in its initial state demonstrated the lowest voltage, half that of tantalum and niobium. After oxidation the (titanium)-(cobaltchromium alloy) and (tantalum)-(cobalt-chromium alloy) pairs showed increased voltages, while with niobium, there was practically no change. It should be noted that for the tantalum pair, the potential susceptibility to corrosion after anodic oxidation was higher because of the voltage and explained by the essential change of tantalum electrode potential in a positive direction, while the cobalt-chromium alloy electrode potential was constant.

Table 2. Voltages between (metal)-(cobalt-chromium alloy) pairs.

\begin{tabular}{cll}
\hline \multirow{2}{*}{ Material } & \multicolumn{1}{c}{ State } & $U / \mathrm{V}$ \\
\hline $\mathrm{Ti}$ & initial state & 0.067 \\
& after oxidation & 0.325 \\
$\mathrm{Nb}$ & initial state & 0.167 \\
& after oxidation & 0.122 \\
$\mathrm{Ta}$ & initial state & 0.130 \\
& after oxidation & 0.459 \\
\hline
\end{tabular}

The analysis of corrosion current, which indicates the rate of metal dissolution, shows that the tantalum after passivation considerably surpassed niobium and especially titanium in its resistance to electrochemical destruction (Table 3). The corrosion rate of niobium, both with its native oxide film and after anodic oxidation, was almost an order of magnitude below that of titanium (in the oxide of which defects are more likely to be present).

Table 3. Corrosion current densities of (metal)-(cobaltchromium alloy) pairs.

\begin{tabular}{clc}
\hline Material & \multicolumn{1}{c}{ State } & $\mathrm{J} / 10^{-2} \mathrm{~A} \mathrm{~m}^{-2}$ \\
\hline $\mathrm{Ti}$ & initial state & 8.041 \\
& after oxidation & 1.105 \\
$\mathrm{Nb}$ & initial state & 1.910 \\
& after oxidation & 0.136 \\
$\mathrm{Ta}$ & initial state & 1.023 \\
& after oxidation & $<0.01$ \\
\hline
\end{tabular}

It is important to note that tantalum and niobium oxides are able to block the corrosion process. If the implant is an anode, the surface oxide film limits metal ion diffusion through the oxide. If the implant is a cathode, the oxide with its very high electrical resistance essentially blocks the electron current and stops reduction processes near the cathode.

The preparation of implants using niobium and tantalum together will not activate electrochemical corrosion processes because the voltage of the tantalum-niobium pair is near to zero.

The oxides of tantalum and niobium are able to selfrestore their electrical resistance when damaged and even increase their own thicknesses according to the degree of environmental activity. Such self-regulating systems, comprising notably metal $(\mathrm{Nb}, \mathrm{Ta})$-oxide, are therefore extremely effective for use as implants. 
The passivation of metal by the forced growth of an oxide on its surface has been demonstrated to provide a more effective barrier against corrosion for niobium and tantalum compared with titanium, and essentially surpasses the passivation due to the formation of a natural surface oxide film by contact with the air.

The dependence of the electrode potential $E$ on the thickness of the surface oxide film is presented in Fig. 1. As the oxide thickness due to thermal oxidation is determined by the duration of the process at constant temperature, and that due to anodic oxidation by the galvanic cell voltage (eqn 1) these parameters were used instead of oxide thickness on the figures. The niobium electrode potential tends to negative values during thermal oxidation. Therefore, the corrosion activity of a niobium implant after such passivation will increase. However, anodic oxidation shifts the niobium potential to electropositive, thereby augmenting its passivity. This result is believed to be due to the heterogeneity of the
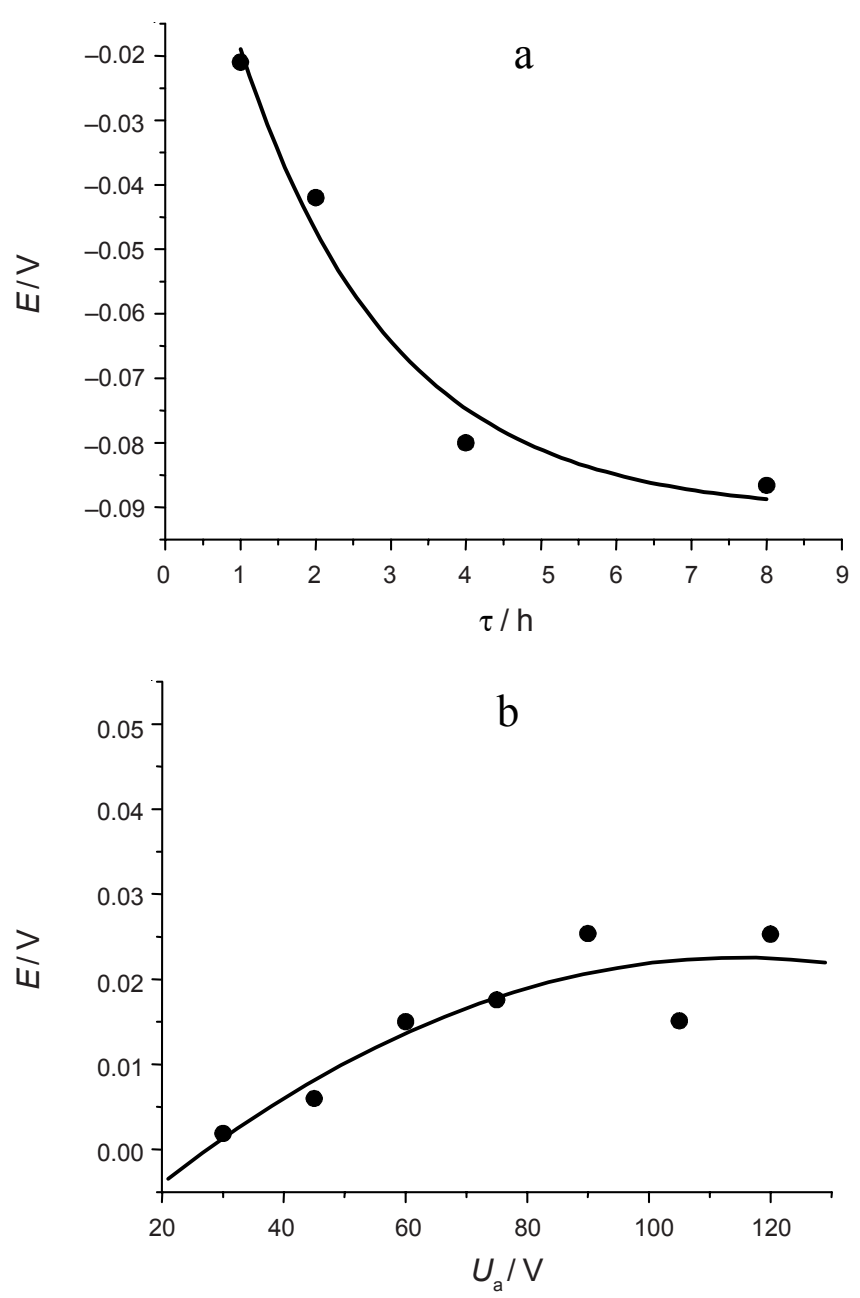

Figure 1. Dependence of electrode potential $E$ of niobium upon thickness of oxide film made by thermal (a) and anodic (b) oxidation. Lines are heuristic exponential or polynomial fits. thermal niobium oxide, as shown for example, by the display of interference colours on the surface after oxidation. During the thermal formation of crystalline oxide, the increase of its thickness results in increased internal mechanical stresses in the film and the appearance of microfissures. On the other hand, the amorphous oxide formed by anodic oxidation is able to sustain considerable deformation and remains pore-free for all investigated thicknesses.

The behaviour of the voltage of the (niobium)(cobalt-chromium alloy) galvanopair (Fig. 2) was different according to whether the metal was passivated via thermal or anodic oxidation. The niobium electrode potential tended to the value of the cobalt-chromium alloy electrode and hence showed a decreased potential difference upon thermal oxidation. During the anodic oxidation of niobium, however, the potential went electropositive and increased the voltage between niobium and the cobalt-chromium alloy. This evident increase of disequilibrium upon contact
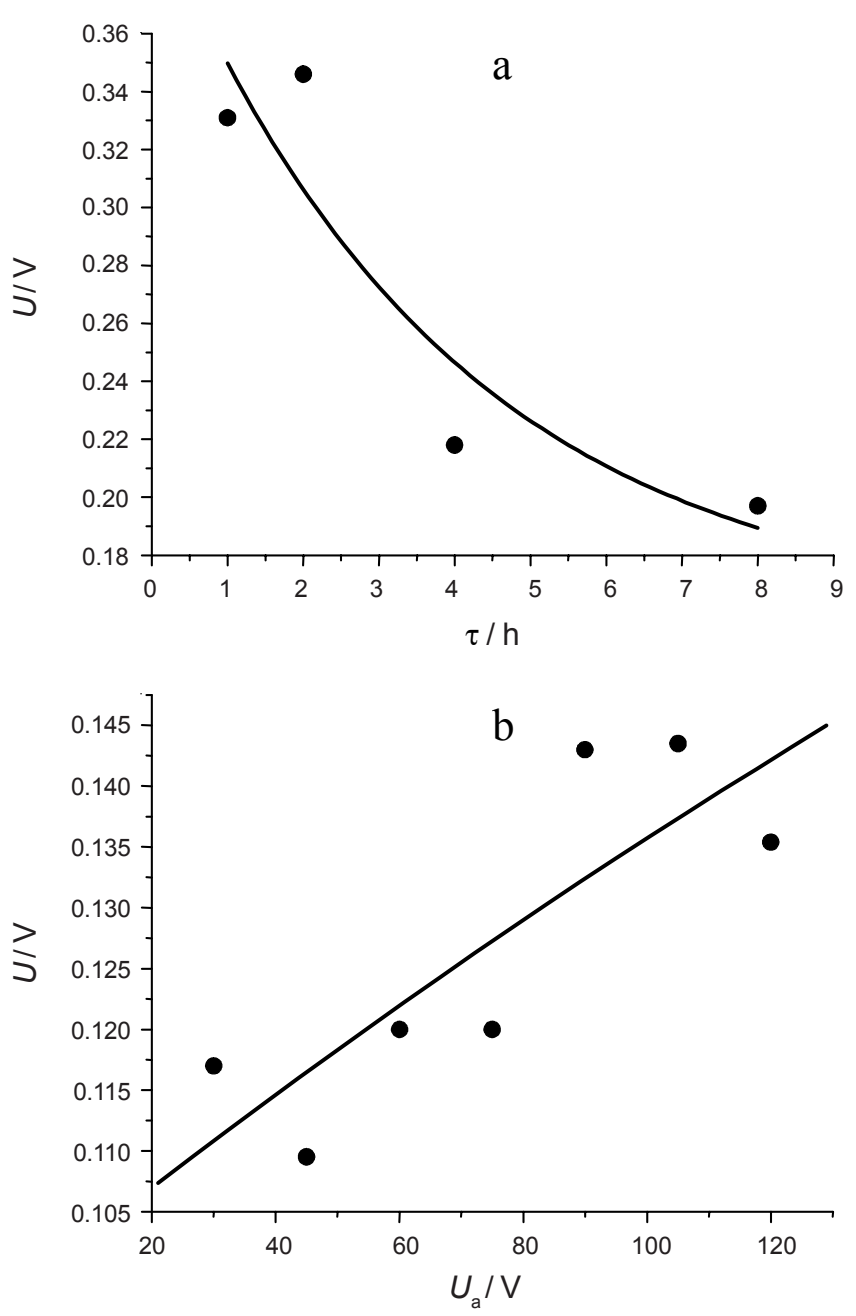

Figure 2. Dependence of the voltage $U$ of the (niobium)-(cobaltchromium alloy) galvanopair on the thickness of niobium surface oxide made by thermal (a) and anodic (b) oxidation. 
of the two metals potentially increases the corrosion of the pair. But the rate of relaxation of this nonequilibrium pair, i.e. the speed of the electrochemical corrosion process, is determined from the analysis of currents shown in Fig. 3.
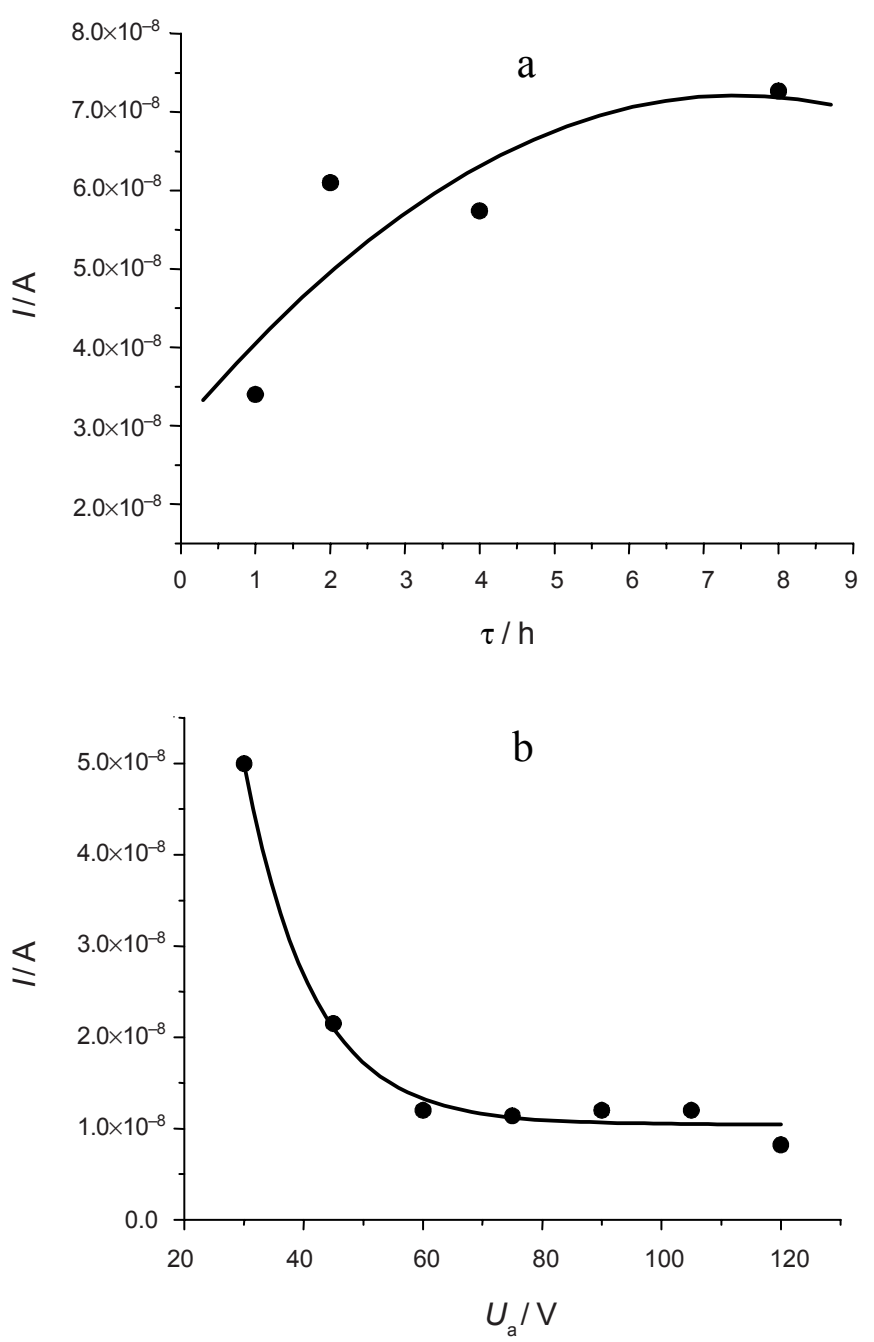

Figure 3. Dependence of corrosion currents $I$ for the (niobium)(cobalt-chromium alloy) galvanopair upon thickness of niobium surface oxide made by thermal (a) and anodic (b) oxidation.

The increase of oxide thickness during the thermal oxidation process of niobium is accompanied by an increase of corrosion current density. The decrease of passivation coating quality results from the presence of microdefects, which increase with its thickness. After anodic oxidation, the quality of metal passivation connected with the growth of the oxide permanently increases, as shown by the decrease in corrosion current. A limiting factor in the corrosion process is the diffusion transfer of metal and oxygen ions through the oxide film on the niobium surface. The oxide thickness increase slows diffusion and, therefore, the whole corrosion process. However, at greater thicknesses of the amorphous oxide film (up to $t=0.35 \mu \mathrm{m}$ ), crystallization processes are activated. The appearance of crystalline oxide in the metal-oxide interphase leads to amorphous oxide destruction, as shown by the formation of cracks and its partial separation from the metal [16].

\section{CONCLUSIONS}

The optimization of the anodic oxide thickness is necessary for prevention of the crystallization process. The experimental data demonstrates that a thickness in the range $t=0.13-0.25 \mu \mathrm{m}$ is optimal. At such an oxide thickness, on the one hand, there is considerable inhibition of the corrosion process in comparison with the natural oxide film. On the other hand, it eliminates the risk of crystallization activation in the amorphous oxide and the oxide preserves its defect-free state.

Passivation of implants made of niobium, tantalum and titanium is best achieved by anodic oxidation of their surfaces. It ensures that a flawless amorphous oxide will be formed, unlike the crystalline oxide that is formed by thermal oxidation. Crystalline oxide is prone to mechanical stresses that lead to microfissures, porosity and inhomogeneous thickness of the oxide coating. An optimally thick anodic oxide transforms medical implants into a passive state, reducing the effects of corrosion.

\section{REFERENCES}

1. Surov, O.N. Dental Prosthetic Implants, p. 208. Moscow: Meditsina (1993) (in Russian).

2. Ramsden, J.J., Allen, D.M., Stephenson, D.J., Alcock, J.R., Peggs, G.N., Fuller, G. \& Goch, G. The design and manufacture of biomedical surfaces. Annals CIRP 56/2 (2007) 687-711.

3. Bayrachniy, B.I. \& Andruschenko, F.K. Electrochemistry of Vent Metals, p. 208. Kiev-Kharkov: Vyschaya Shkola (1985), (in Russian).

4. Schider, S. \& Bildstein, H. Tantalum and niobium as potential prosthetic materials. In: Advances in Biomaterials (ed. G.D. Winter, D.F. Gibbons and H. Plenk, Jr.), pp. 13-20. New York: Wiley (1980).

5. Liu, X., Huang, A., Ding, C. \& Chua, P.K. Bioactivity and cytocompatibility of zirconia $\left(\mathrm{ZrO}_{2}\right)$ films fabricated by cathodic arc deposition. Biomaterials 27 (2006) 3904-3911.

6. Kay, J.F. A new concept for noncement fixation of orthopaedic devices. Techniques in Orthopaedics 2 (1987) 1-6.

7. Mamalis, A.G., Ramsden, J.J., Grabchenko, A.I., Lytvynov, L.A., Filipenko, V.A. \& Lavrynenko, S.N. A novel concept for the manufacture of individual sapphire-metallic hip joint endoprostheses. J. Biol. Phys. Chem. 6 (2006) 113-117.

8. Ungersboeck, A., Geret, V., Pohler, O et al. Tissue reaction to bone plates made of pure titanium: a prospective, quantitative clinical study. J. Mater. Sci. 6 (1995) 223-229.

9. Brunski, J.B., Puleo, D.A. \& Nanci, A. Biomaterials and biomechanics of oral and maxillofacial implants: current status and future developments. Int. J. Oral Maxillofacial Implants 1 (2000) 15-46. 
10. Cook, S.D., Kay, J.F., Thomas, K.A. \& Jarcho, M. Interface mechanics and histology of titanium and hydroxyapatitecoated titanium for dental implant applications. Int. J. Oral Maxillofacial Implants 2 (1987) 15-22.

11. Matsui, Y., Ohno, K., Michi, K. \& Yamagata, K. Experimental study of high-velocity flame-sprayed hydroxyapatite coated and noncoated titanium implants. Int. J. Oral Maxillofacial Implants 9 (1994) 397-404.

12. Albrektsson, T., Eriksson, A.R., Friberg, B., Lekholm, U. et al. Histologic investigations on 33 retrieved Nobelpharma implants. Clin. Mater. 12 (1993) 1-9.

13. Wong, M., Eulenberger, J., Schenk, R. \& Hunziker, E. Effect of surface topology on the integration of implant materials in trabecular bone. J. Biomed. Mater. Res. 29 (1995) 1567-1575.

14. Kutsevlyak, V.I., Starikova, S.L. \& Starikov, V.V. Features of electrochemical interaction between of implant and prosthetic materials. Modern Dentistry (Kyiv) 4 (2005) 130-132 (in Russian).

15. Larsson, Cc., Thomsen, P., Lausmaa, J., Ridahl, M., Kasemo, B. $\&$ Ericson L.E. Bone response to surface modified titanium implants: studies on electropolished implants with different oxide thicknesses and morphology. Biomaterials 15 (1994) 106-274.

16. Odynets, L.L. \& Orlov, V.M. Anodic Oxide Films, p. 200. Leningrad: Nauka (1990) (in Russian). 